\title{
REAL-TIME EMBEDDED SLIDING MODE OBSERVER FOR QUADCOPTER UAVs
}

\author{
A. R. FIRDAUS ${ }^{1}$, and M. O. TOKHI ${ }^{2}$ \\ ${ }^{1}$ Department of Automatic Control and Systems Engineering, \\ The University of Sheffield, United Kingdom \\ ${ }^{2}$ School of Engineering, London South Bank University, \\ United Kingdom.
}

\begin{abstract}
Sliding mode observer (SMO) as a nonlinear and robust observer, is believed to be able to provide all required states information for control process of quadcopter UAVs. In this paper, a comparative assessment through numerical simulation is conducted between SMO and Extended Kalman Filter (EKF) to demonstrate the performance of both estimators. The results obtained demonstrate good performance of SMO in dealing with noise and uncertainty. Furthermore, experiments are carried out to validate the performance of SMO in real-time. The results show that estimated states can track true states fast with small estimation steady state errors and the observer estimates the unmeasured states smoothly.
\end{abstract}

Keywords: Nonlinear systems; quadcopter; sliding mode observer; extended Kalman filter.

\section{Introduction}

The states information of a controlled process plays a critical role in controlling quadcopter UAVs. Although information on states can be obtained accurately with high precision sensor, such as VICON motion capture as conducted by Satici et al., ${ }^{1}$ the system will become more expensive. Therefore, the use of an observer can be a solution to reduce cost for acquiring unmeasured states. Previous works have considered the use of different types of observer methods in quadcopter UAVs. These include sliding mode observer (SMO), ${ }^{2}$ Thau observer ${ }^{3}$ and Kalman filter. ${ }^{4}$ The results show that such methods can enhance the controller performance. However, this does not mean these methods are free of shortcomings. A comparative study between SMO and Kalman filter (KF) was carried out by. ${ }^{5}$ They pointed out that $\mathrm{SMO}$ is more robust than $\mathrm{KF}$ in terms of parameter uncertainty 
and noise cancellation. Thau observer may not deal with model error of system. ${ }^{6}$ Although SMO outperforms KF and Thau observer, few studies have been carried out to demonstrate the performance of SMO experimentally for rotorcraft-based UAVs.

This paper proposes application of sliding mode observer with implementation in real-time embedded system of quadcopter UAV. Moreover, numerical simulations of the comparison of SMO and Extended Kalman Filter (EKF) performance are presented in estimating the states of quadcopter dynamic system subject to uncertainty and noise disturbance. The rest of the paper is structured as follows: Section II describes dynamic model of quadcopter, Section III presents flight control and observer design, Section IV results and discussion. The paper is concluded in Section V.

\section{Quadcopter Dynamic System}

The dynamical model of quadcopter cited from ${ }^{7}$ can be expressed as

$$
\begin{gathered}
\ddot{x}=\frac{1}{m}(c \phi s \theta c \psi+s \phi s \psi) u_{1}-\frac{k_{1}}{m} \dot{x} \\
\ddot{y}=\frac{1}{m}(c \phi s \theta s \psi-s \phi c \psi) u_{1}-\frac{k_{2}}{m} \dot{y} \\
\ddot{z}=\frac{1}{m} c \phi c \theta u_{1}-g-\frac{k_{3}}{m} \dot{z} \\
\ddot{\phi}=\dot{\theta} \dot{\psi}\left(\frac{I_{y}-I_{z}}{I_{x}}\right)+\frac{l}{I_{x}} u_{2}-\frac{k_{4} l}{I_{x}} \dot{\phi} \\
\ddot{\theta}=\dot{\psi} \dot{\phi}\left(\frac{I_{z}-I_{x}}{I_{y}}\right)+\frac{l}{I_{y}} u_{3}-\frac{k_{5} l}{I_{y}} \dot{\theta} \\
\ddot{\psi}=\dot{\phi} \dot{\theta}\left(\frac{I_{x}-I_{y}}{I_{z}}\right)+\frac{C}{I_{z}} u_{4}-\frac{k_{6}}{I_{z}} \dot{\psi}
\end{gathered}
$$

where $m, g$, and $l$ represent the total mass of the rotorcraft, gravity acceleration, and the distance of each rotor to centre of mass of rotorcraft respectively. The angles $(\phi, \theta, \psi)$ represent roll, pitch, and yaw, while $s, c$, and $t$ represent sine,cos, and tangent respectively. The movement of roll, pitch and yaw are bounded as: $(-\pi / 2<\phi<\pi / 2),(-\pi / 2<\theta<\pi / 2)$ and $(-\pi<\psi<\pi)$ respectively. $k_{i}$ represent the coefficients of drag and positive constants, while $I_{x}, I_{y}, I_{z}$ denote the inertia of rotorcraft in $x, y, z$ axes respectively. The control inputs of quatcopter are represented by $u_{1}$ for total thrust of rotorcraft in $z$-axis, and $u_{2}, u_{3}$, and $u_{4}$ for roll, pitch, and yaw respectively.

\section{Control and Observer Design}

\subsection{Sliding mode control}

The desired trajectories are represented as $x_{d}$ and $\dot{x}_{d}$, whereas the tracking errors are $e=x-x_{d}$ and $\dot{e}=\dot{x}-\dot{x_{d}}$, where $x$ and $\dot{x}$ represent state and 
derivative with respect to time of state. The sliding mode manifold $(\sigma)$ is expressed as

$$
\sigma(t)=s e+\dot{e}
$$

The variable $s$ is the slope of sliding surface. Hence, the sliding manifolds for quadcopter UAVs are expressed as

$$
\begin{gathered}
\sigma_{\phi}=s_{\phi}\left(\phi-\phi_{d}\right)+\left(\dot{\phi}-\dot{\phi}_{d}\right) \\
\sigma_{\theta}=s_{\theta}\left(\theta-\theta_{d}\right)+\left(\dot{\theta}-\dot{\theta}_{d}\right) \\
\sigma_{\psi}=s_{\psi}\left(\psi-\psi_{d}\right)+\left(\dot{\psi}-\dot{\psi}_{d}\right) \\
\sigma_{z}=s_{z}\left(z-z_{d}\right)+\left(\dot{z}-\dot{z}_{d}\right) \\
\sigma_{x}=s_{x}\left(x-x_{d}\right)+\left(\dot{x}-\dot{x_{d}}\right) \\
\sigma_{y}=s_{y}\left(y-y_{d}\right)+\left(\dot{y}-\dot{y}_{d}\right)
\end{gathered}
$$

The tracking errors will asymptotically converge to zero if the conditions $\sigma(x, t)=0$ and $\sigma(\dot{x}, t)=0$ are fulfilled at every $t \geq t_{0}$. The sign function is smoothened using the approximation $\operatorname{sign}(\sigma) \approx \operatorname{sigmoid}(\sigma)$.

Considering the dynamic model system in equation (1)-(6), sliding surface in equation (8)-(13), and sliding condition $\dot{\sigma}(x, t)=0$, the control input can be expressed as

$$
\begin{gathered}
u_{1}=\frac{m}{c \phi c \theta}\left(-k_{z} \tanh \left(\sigma_{z}\right)-k_{z} \sigma_{z}-\frac{k_{3} \dot{z}}{m}+\ddot{z_{d}}-s_{z} e_{\dot{z}}+g\right) \\
u_{2}=\frac{I_{x}}{l}\left(-k_{\phi} \tanh \left(\sigma_{\phi}\right)-k_{\phi} \sigma_{\phi}-\frac{\dot{\theta} \dot{\psi}}{I_{x}}\left(I_{y}-I_{z}\right)-\frac{k_{4} l}{I_{x}} \dot{\phi}+\ddot{\phi}_{d}-s_{\phi} e_{\dot{\phi}}\right) \\
u_{3}=\frac{I_{y}}{l}\left(-k_{\theta} \tanh \left(\sigma_{\theta}\right)-k_{\theta} \sigma_{\theta}-\frac{\dot{\phi} \dot{\psi}}{I_{y}}\left(I_{z}-I_{x}\right)-\frac{k_{5} l}{I_{y}} \dot{\theta}+\ddot{\theta_{d}}-s_{\theta} e_{\dot{\theta}}\right) \\
u_{4}=\frac{I_{z}}{C}\left(-k_{\psi} \tanh \left(\sigma_{\psi}\right)-k_{\psi} \sigma_{\psi}-\frac{\dot{\phi} \dot{\theta}}{I_{z}}\left(I_{x}-I_{y}\right)-\frac{k_{6}}{I_{z}} \dot{\psi}+\ddot{\psi}_{d}-s_{\psi} e_{\dot{\psi}}\right)
\end{gathered}
$$

The calculations of desired pitch and roll angles with $x$ and $y$ axes errors are shown as

$$
\begin{gathered}
\phi_{d}=-\operatorname{atan}\left(\frac{\left(-k_{y} \tanh \left(\sigma_{y}\right)-k_{y} \sigma_{y}-\frac{k_{2} \dot{y}}{m}+\ddot{y_{d}}-s_{y} e_{\dot{y}}\right) c \phi c \theta}{-k_{z} \tanh \left(\sigma_{z}\right)-k_{z} \sigma_{z}-\frac{k_{3} \dot{z}}{m}+\ddot{z}_{d}-s_{z} e_{\dot{z}}+g}\right) \\
\theta_{d}=\operatorname{atan}\left(\frac{\left(-k_{x} \tanh \left(\sigma_{x}\right)-k_{x} \sigma_{x}-\frac{k_{1} \dot{x}}{m}+\ddot{x_{d}}-s_{x} e_{\dot{x}}\right) c \phi c \theta}{-k_{z} \tanh \left(\sigma_{z}\right)-k_{z} \sigma_{z}-\frac{k_{3} \dot{\dot{z}}}{m}+\ddot{z}_{d}-s_{z} e_{\dot{z}}+g}\right)
\end{gathered}
$$

\subsection{Sliding mode observer}

States vector $x(t)$ is defined as:

$x_{1}=\phi ; x_{2}=\dot{\phi} ; x_{3}=\theta ; x_{4}=\dot{\theta} ; x_{5}=\psi ; x_{6}=\dot{\psi}$

$x_{7}=z ; x_{8}=\dot{z} ; x_{9}=x ; x_{10}=\dot{x} x_{11}=y ; x_{12}=\dot{y}$

In this case, the measured states of the rotorcraft are $\operatorname{roll}(\phi), \operatorname{pitch}(\theta)$ and yaw $(\psi)$ angles, and movements in $x, y, z$ axes. Thus, in light of previous work in, ${ }^{8}$ the SMO for the quadcopter model is presented as

$$
\begin{gathered}
\dot{\hat{x}}_{1}=-\alpha_{1} \tilde{x}_{1}+\hat{x}_{2}-K_{1} \tanh \left(\tilde{x}_{1}\right) \\
\dot{\hat{x}}_{2}=-\alpha_{2} \tilde{x}_{1}+\hat{x}_{4} \hat{x}_{6}\left(\frac{I_{y}-I_{z}}{I_{x}}\right)-\frac{k_{4} l \hat{x}_{2}}{I_{x}}-K_{2} \tanh \left(\tilde{x}_{1}\right)
\end{gathered}
$$




$$
\begin{gathered}
\dot{\hat{x}}_{3}=-\alpha_{1} \tilde{x}_{3}+\hat{x}_{4}-K_{1} \tanh \left(\tilde{x}_{3}\right) \\
\dot{\hat{x}}_{4}=-\alpha_{2} \tilde{x}_{3}+\hat{x}_{2} \hat{x}_{6}\left(\frac{I_{z}-I_{x}}{I_{y}}\right)-\frac{k_{5} l \hat{x}_{4}}{I_{y}}-K_{2} \tanh \left(\tilde{x}_{3}\right) \\
\dot{\hat{x}}_{5}=-\alpha_{1} \tilde{x}_{5}+\hat{x}_{6}-K_{1} \tanh \left(\tilde{x}_{5}\right) \\
\dot{\hat{x}}_{6}=-\alpha_{2} \tilde{x}_{5}+\hat{x}_{2} \hat{x}_{4}\left(\frac{I_{x}-I_{y}}{I_{z}}\right)-\frac{k_{6} \hat{x}_{6}}{I_{z}}-K_{2} \tanh \left(\tilde{x}_{5}\right) \\
\dot{\hat{x}}_{7}=-\alpha_{1} \tilde{x}_{7}+\hat{x}_{8}-K_{1} \tanh \left(\tilde{x}_{7}\right) \\
\dot{\hat{x}}_{8}=-\alpha_{2} \tilde{x}_{7}-g-\frac{k_{3} \hat{x}_{8}}{m}-K_{2} \tanh \left(\tilde{x}_{7}\right) \\
\dot{\hat{x}}_{9}=-\alpha_{1} \tilde{x}_{9}+\hat{x}_{10}-K_{1} \tanh \left(\tilde{x}_{9}\right) \\
\dot{\hat{x}}_{10}=-\alpha_{2} \tilde{x}_{9}-\frac{k_{1} \hat{x}_{10}}{m}-K_{2} \tanh \left(\tilde{x}_{9}\right) \\
\dot{\hat{x}}_{11}=-\alpha_{1} \tilde{x}_{11}+\hat{x}_{12}-K_{1} \tanh \left(\tilde{x}_{11}\right) \\
\dot{\hat{x}}_{12}=-\alpha_{2} \tilde{x}_{11}-\frac{k_{2} \hat{x}_{12}}{m}-K_{2} \tanh \left(\tilde{x}_{11}\right)
\end{gathered}
$$

where $\tilde{x}=\hat{x}-x$ is the estimation error of states, $\hat{x}, \alpha_{i}$ and $K_{i}$ represent estimated states, constants, and positive constants respectively.

\section{Results and Discussion}

\subsection{Simulation results}

In this section a set of simulation exercises are presented to examine and demonstrate the performance of SMO approach and EKF in estimating the unmeasured states of the quadcopter. Disturbances and uncertainties such as e.g. air drag, white Gaussian noise, and parameters mismatch have been incorporated into the simulation to demonstrate the robustness of the observer.

Table 1. Quadcopter parameters.

\begin{tabular}{cccc}
\hline Variables & Observer model & Plant & Units \\
\hline$m$ & 1.790 & 1.790 & $\mathrm{~kg}$ \\
$l$ & 0.290 & 0.290 & $\mathrm{~m}$ \\
$I_{x}$ & 0.02615 & 0.01318 & $\mathrm{Ns} 2 / \mathrm{rad}$ \\
$I_{y}$ & 0.02735 & 0.01284 & $\mathrm{Ns} 2 / \mathrm{rad}$ \\
$I_{z}$ & 0.04538 & 0.02108 & $\mathrm{Ns} 2 / \mathrm{rad}$ \\
$k_{i}(i=1,2,3)$ & 0.37 & 0.37 & $\mathrm{Ns} / \mathrm{m}$ \\
$k_{i}(i=4,5,6)$ & 0.0005 & 0.0005 & $\mathrm{Ns} 2 / \mathrm{rad}$ \\
$g$ & 9.81 & 9.81 & $\mathrm{~m} / \mathrm{s} 2$ \\
\hline
\end{tabular}

The moment of inertia (Turnigy H.A.L frame), mass, and length of quadcopter arms are real parameters obtained by measurement. Furthermore, to demonstrate the robustness of the observer, parameters mismatch between observer model and plant was used. Moment of inertia of LJI frame was applied for plant parameters. All parameters used in the simulation are shown 
in Table 1. Noise disturbance to measured states are 10dB for roll and pitch angle, and $37 \mathrm{~dB}$ for yaw angle, $x, y$, and $z$ movement. Suitable values of SMO parameters were selected, such as $\alpha_{1}=20, \alpha_{2}=7.2, K_{1}=0.1$ and $K_{2}=50$, while measurement and process noise covariance of EKF estimator are $R=0.001$ and $Q=0.1$ respectively.

The simulation results obtained are shown in Figs. $1-12$

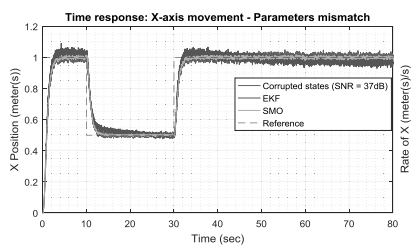

Fig. 1. $x$-axis movement.

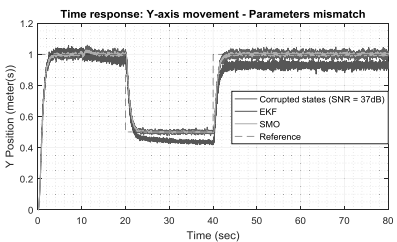

Fig. 3. $y$-axis movement.

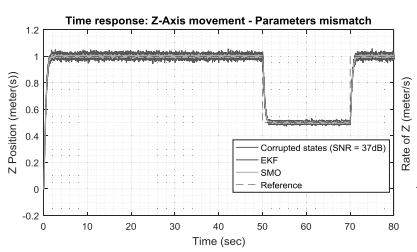

Fig. 5. $z$-axis movement.

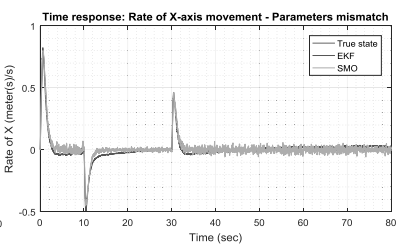

Fig. 2. Rate of $x$-axis.

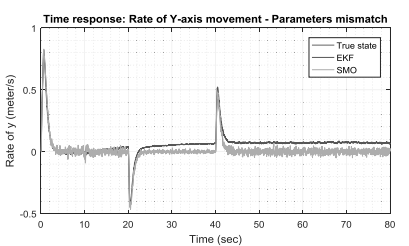

Fig. 4. Rate of $y$-axis.

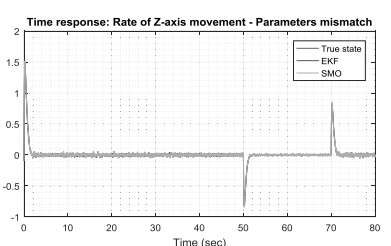

Fig. 6. Rate of $z$-axis.

Generally, although in the presence of noise and parameter mismatch appeared between observer and real plant, both observers worked in estimating unmeasured states of the quadcopter. Comparative results in Figs. 1 - 4 show that EKF had higher steady state estimation errors than SMO, by $\pm 6 \%$ in $y$-movement, $\pm 3 \%$ in $x$-movement, $\pm 2 \%$ in rate of $x$-movement, and $\pm 4 \%$ in rate of $y$-movement. These simulations show that, although 


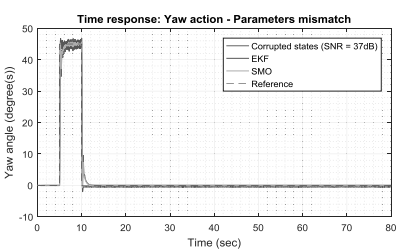

Fig. 7. Yaw action.

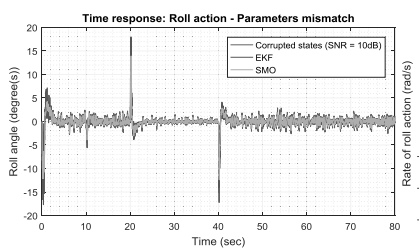

Fig. 9. Roll action.

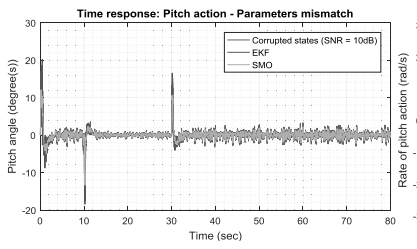

Fig. 11. Pitch action.

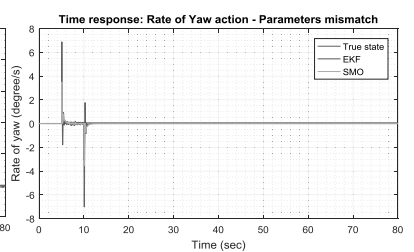

Fig. 8. Rate of Yaw action.

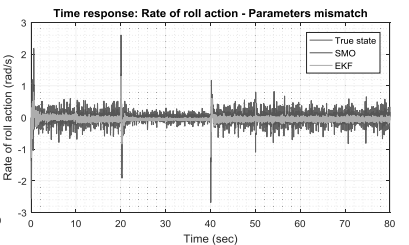

Fig. 10. Rate of Roll action.

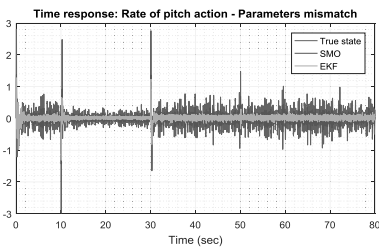

Fig. 12. Rate of Pitch action.

the states were corrupted with high power of noise and parameters mismatch, the sliding mode observer provided adequate performance. Furthermore, SMO gave appropriate states information so that SMC was still able to maintain the stability of movement of the quadrotor as seen in Figs. 1, 3, 5, 7, 9, and 11. In other words, SMO contributed to better performance of controller than EKF particularly in controlling $x$ and $y$ movement of the rotor-craft. Parameter mismatch did not have significant effect on performance of the observers.

\subsection{Experimental results}

Considering the simulation results, the performance of SMO was validated experimentally to show how this observer can deal with uncertainty and noise in real time application. The same quadcopter and observer parameters as in the numerical simulations were selected for this experiment. The experimental results obtained are shown in Figs. $13-20$. 


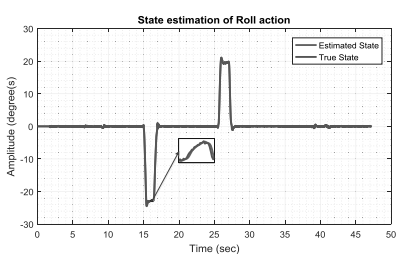

Fig. 13. Roll action.

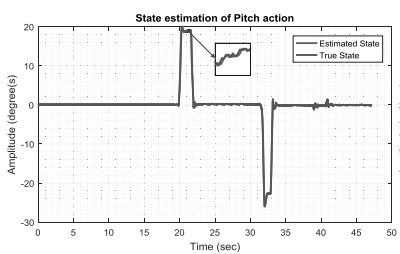

Fig. 15. Pitch action.

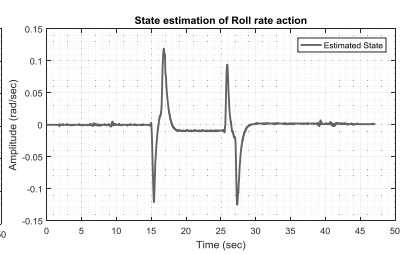

Fig. 14. Rate of Roll action.

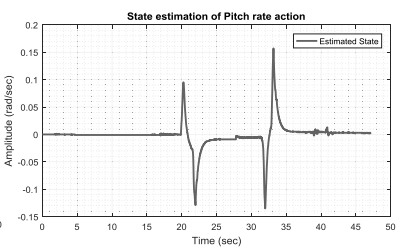

Fig. 16. Rate of Pitch action.
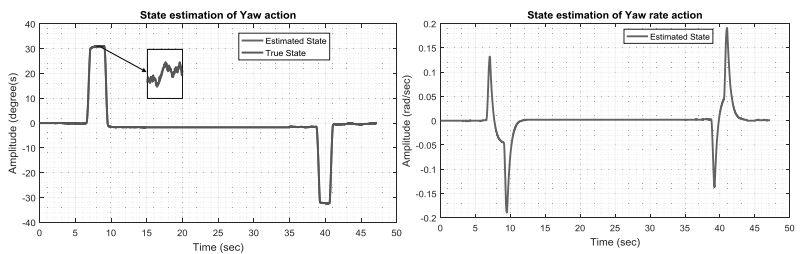

Fig. 17. Yaw action.

Fig. 18. Rate of Yaw action.

The experimental results show that generally estimated states can track true states fast with small estimation steady state errors. Particularly, the steady state estimation error is very small near to zero $(\sim 0)$ in tracking the true state of $z$-movement. Furthermore, the measurement noise from IMU sensors gave no significant effect to estimation process by showing smooth estimation of unmeasured states as shown in Figs. 14, 16, 18, and 20.

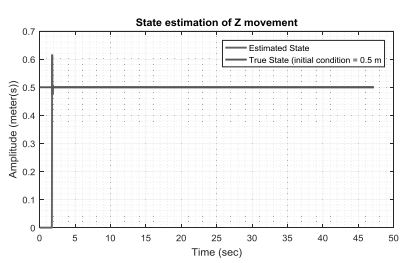

Fig. 19. $z$-axis movement.

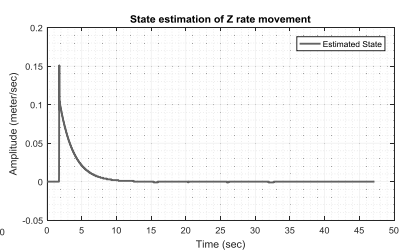

Fig. 20. Rate of $z$ - axis. 


\section{Conclusion}

Comparative studies have been conducted between SMO and EKF in numerical simulations to demonstrate the advantages of SMO method in estimating unmeasured states of a quadcopter. From the results achieved it can be concluded that SMO scheme can provide robustness compared to EKF in dealing with nonlinearity, parameter mismatch, and disturbance noise by enabling convergence of the estimated states to the true values.

Sliding mode observer has been validated in real-time embedded system and the results show that such observer performs well by showing smooth estimation for unmeasured states and cancelling the effect of measurement noise from IMU sensor in the estimation process.

The observer can thus be a promising method for real-time control of quadcopter and other nonlinear systems. Future research will focus on working on $x$ and $y$ sensors and estimating the derivative of such states for better autonomous system quadcopter.

\section{Acknowledgment}

This work is supported by LPDP - Indonesia Endowment Fund for Education, and Politeknik Negeri Batam.

\section{References}

1. A. C. Satici, H. Poonawala and M. W. Spong, Access, IEEE 1, 79 (2013).

2. X. Wang and B. Shirinzadeh, Nonlinear Dynamics 80, 1463 (2015).

3. Z. Cen, H. Noura and Y. A. Younes, Robust fault estimation on a real quadrotor uav using optimized adaptive thau observer, in 2013 International Conference on Unmanned Aircraft Systems, ICUAS 2013 - Conference Proceedings, 2013.

4. S. A. Belokon', Y. N. Zolotukhin, K. Y. Kotov, A. S. Mal'tsev, A. Nesterov, V. Y. Pivkin, M. A. Sobolev, M. N. Filippov and A. P. Yan, Optoelectronics, Instrumentation and Data Processing 49, 536 (2013).

5. F. Chen and M. Dunnigan, Comparative study of a sliding mode observer and kalman filters for full state estimation in an induction machine, in IEE Proceedings-Electric Power Applications, 2002.

6. D. Schroder, Intelligent observer and control design for nonlinear systems (Springer, Berlin, Germany, 2000).

7. E.-H. Zheng, J.-J. Xiong and J.-L. Luo, ISA transactions 53, 1350 (2014).

8. J.-J. E. Slotine, J. K. Hedrick and E. a. Misawa, On sliding observers for nonlinear systems, in 1986 American Control Conference, 1986. 